Research Article

\title{
Clinical and Mortality Risk Factors in Bloodstream Infections with Carbapenem-Resistant Enterobacteriaceae
}

\author{
Xiaopeng Li and Huan Ye \\ Department of Infectious Diseases, Fuxing Hospital, Capital Medical University, Beijing, China \\ Correspondence should be addressed to Huan Ye; yedahuan01@126.com
}

Received 21 July 2017; Revised 22 September 2017; Accepted 27 September 2017; Published 12 December 2017

Academic Editor: José Ramón Blanco

Copyright (c) 2017 Xiaopeng Li and Huan Ye. This is an open access article distributed under the Creative Commons Attribution License, which permits unrestricted use, distribution, and reproduction in any medium, provided the original work is properly cited.

Objective. To investigate the risk factors underlying the occurrence and mortality of bloodstream infections (BSIs) with carbapenem-resistant Enterobacteriaceae (CRE). Methods. Medical information was retrospectively analyzed from 148 cases of patients with Enterobacteriaceae BSIs at a medical center in China, between 2013 and 2015. Results. The 30-day mortality rate in the CRE group was $65.4 \%$. Indwelling urethral catheterization, admission to the ICU, use of antibiotics within 30 days, and BSIs from the respiratory system were associated with CRE BSIs. Lung infection, abdominal infection, central venous catheterization, and use of hormones within 30 days were associated with mortality. Conclusion. The 30-day mortality rate of CRE BSIs was high. Lung infections, abdominal infections, central venous catheterization, and use of hormones within 30 days increased the mortality rate of Enterobacteriaceae BSIs.

\section{Introduction}

Recently, infections with drug-resistant bacterial strains have increased which poses a challenge for anti-infection treatments in the clinical context and has become a serious problem in the field of public health. The incidence of bloodstream infections is also increasing and has become a major cause of the occurrence of infectious diseases and deaths worldwide [1]. Bloodstream infections with multidrugresistant Enterobacteriaceae account for $71.5 \%$ of all bloodstream infections with multidrug-resistant bacterial strains [2]. Carbapenem antibiotics are typically one of the most effective drug classes for the treatment of Enterobacteriaceae infections. However, carbapenem-resistant Enterobacteriaceae (CRE) has been identified recently within all Enterobacteriaceae spp. The CHINET monitoring results show that Klebsiella pneumoniae accounts for the majority of cases of CRE infections among the Enterobacteriaceae spp., with drug resistance rates to imipenem and meropenem $>10 \%$ [3]. Studies have shown that the mortality rate is significantly higher for CRE infections than for carbapenem-sensitive Enterobacteriaceae infections [4].
This study was a retrospective analysis of medical information on bloodstream infections with Enterobacteriaceae (mainly Klebsiella pneumoniae, Escherichia coli, Enterobacter cloacae, and Enterobacter aerogenes) with intent to identify the risk factors and prognosis for CRE bloodstream infections.

\section{Materials and Methods}

2.1. Study Subjects. A total of 148 cases of bloodstream infections with Enterobacteriaceae that occurred during the period between January 2013 and October 2015 in Fuxing Hospital of Capital Medical University, China, were selected. Clinical information from the patients, including demographic data, underlying diseases, pathogen drug resistance and drug sensitivity analyses, history of medical institution admission, level of care (ward versus ICU), history of invasive surgery, and prognosis, was recorded and analyzed. The characteristics of each case were collected from the electronic medical record database, and the files of all patients were complete.

Inclusion criteria: On the day of collection of a positive blood culture specimen, the patient had a temperature $\geq 38^{\circ} \mathrm{C}$ 
or $<36^{\circ} \mathrm{C}$ in combination with chills, and the multiple blood culture results showed the same type of bacteria. Exclusion criteria: (1) blood culture test results showing two or more types of bacteria and (2) only one blood culture specimen was positive, whereas multiple subsequent blood culture results were negative or identified other types of pathogens.

This study conformed to the standards of medical ethics and was approved by the Ethics Committee of Fuxing Hospital of Capital Medical University.

2.2. Relevant Definitions. The microbiology laboratory in our hospital currently uses an FX200 automatic blood culture instrument (BACTE) and a WalkAway 40 automatic microbiological drug sensitivity analysis instrument (USA). The drug sensitivity results were determined according to the Clinical and Laboratory Standards Institute (CLSI, USA) standards [5]. The definition of CRE was resistance to imipenem, meropenem, or ertapenem [3]. Extensively drugresistant (XDR) bacteria were defined based on nonsusceptibility to all antimicrobial agents except colistin and tigecycline $[6,7]$. Long-term bedbound patients were defined as patients who were bedbound for more than 14 days and could not recover [7].

2.3. Statistical Methods. The data processing and analysis were performed using SPSS 21.0 statistical software. $P<0.05$ indicated that the difference was significant. Measurement data with a normal distribution are expressed using the mean \pm standard deviation. Measurement data with a nonnormal distribution are expressed using the median. Significance testing for differences between groups was performed using the $t$-test (normal distribution) or rank sum test (nonnormal distribution). Significance testing for differences in count data between groups was performed using the $\chi^{2}$ test. The multivariate analysis was performed using logistic regression.

\section{Results}

3.1. Demographic Data and Clinical Characteristics. This study involved a total of 148 cases of bloodstream infections with Enterobacteriaceae. The mean age of the patients was $69.1( \pm 19.2)$ years. Male patients accounted for $50.7 \%$ of the cases, and $20.3 \%$ of the patients were admitted to the ICU. Long-term bedbound patients accounted for $54.1 \%$ of the cases, and $62.2 \%$ of the patients had a medical institution admission history within 3 months. The bloodstream infections mainly originated from the urinary tract $(28.4 \%)$ or respiratory system (25.0\%) or were catheter related $(22.3 \%)$. Patients who had prior use of broad-spectrum antibiotics within 30 days accounted for $43.2 \%$ of the cases. The total average length of hospital stay was $21.7( \pm 12.5)$ days. Patients with CRE infections accounted for $17.6 \%$ of the cases, and patients with XDR Enterobacteriaceae infections accounted for $4.0 \%$ of the cases.

The results of the univariate and multivariate analyses of risk factors related to CRE bloodstream infections are shown in Tables 1 and 2, respectively (the relevant factors listed in the tables were all conditions before the presence of the bloodstream infection). The logistic regression analysis revealed that indwelling urethral catheterization, admission to the ICU, CRE infection of the respiratory system, and prior use of broad-spectrum antibiotics within 30 days were independent risk factors for CRE bloodstream infections.

3.2. Analysis of Risk Factors for Mortality in Bloodstream Infections with Enterobacteriaceae. According to the comparison of the 30-day prognosis between groups, the 30-day mortality rate for bloodstream infections with Enterobacteriaceae was $25.7 \%$. Univariate analysis indicated that community acquired, admission to the ICU, long-term bedbound, anemia, hypoalbuminemia, lung infection, abdominal infection, liver dysfunction, mechanic ventilation, central venous catheterization, indwelling urethral catheterization, sources of bloodstream infection from catheter related and urinary tract, antibiotics within 30 days, combined fungal infection, prior use of glucocorticoids within 30 days, and CRE infection were significantly associated with 30-day risk of mortality (Tables 3). A logistic regression analysis revealed that lung infection, abdominal infection, central venous catheterization, and prior use of glucocorticoids within 30 days were independently associated with mortality (Tables 4).

\section{Discussion}

Currently, the incidence of carbapenemase-producing Enterobacteriaceae is increasing. Data have shown that many countries have interregional spread or an endemic situation [8]. The results of this study showed that the 30-day mortality rate for CRE bloodstream infections reached $65.4 \%$, which was significantly higher than the rate for nonCRE bloodstream infections $(17.2 \%)$ or for CRE-infected patients in literature reports $(26 \%-44 \%)$ [4]. To date, many studies have evaluated risk factors related to bloodstream infections with multidrug-resistant Enterobacteriaceae. For example, factors including admission to the ICU, length of hospital stay, history of use of broad-spectrum antibiotics, such as quinolones and cephalosporins, history of resistant strain colonization, indwelling urethral catheterization, and central venous catheterization have been considered independent risk factors for bloodstream infections with multidrug-resistant Enterobacteriaceae [4, 9]. The analytical results in this study showed that indwelling urethral catheterization, admission to the ICU, prior use of broadspectrum antibiotics within 30 days, and CRE infection of the respiratory system were independent risk factors for CRE bloodstream infections. The first three factors are similar to previous reports. However, what is different from previous reports is that we identified CRE infection of the respiratory system as an independent risk factor for CRE bloodstream infections. Respiratory tract infection was the most common source of bloodstream infection in our study, likely because of a decreased capacity for bacterial clearance resulting from the altered lung tissue in severe chronic obstructive lung disease. 
TABLE 1: Univariate analysis of the clinical characteristics of bloodstream infections caused by CRE and non-CRE.

\begin{tabular}{|c|c|c|c|}
\hline \multirow{2}{*}{ Clinical characteristics } & \multicolumn{2}{|c|}{ Bloodstream infections with Enterobacteriaceae, $n(\%)$} & \multirow{2}{*}{$P$ value } \\
\hline & CRE group $(n=26)$ & Non-CRE group $(n=122)$ & \\
\hline Gender (male) & $16(61.5)$ & $59(48.4)$ & 0.222 \\
\hline Age $>60$ years & $22(84.6)$ & $83(68.0)$ & 0.091 \\
\hline Community acquired & 0 & $30(24.6)$ & $0.005^{*}$ \\
\hline Admission to the ICU & $20(76.9)$ & $10(8.2)$ & $<0.001^{*}$ \\
\hline Long-term bedbound & $19(73.1)$ & $61(50)$ & $0.032^{*}$ \\
\hline Medical institution admission history within 3 months & $17(65.4)$ & $75(61.5)$ & 0.709 \\
\hline \multicolumn{4}{|l|}{ Underlying diseases } \\
\hline Cardiovascular disease & $19(73.1)$ & $54(44.3)$ & $0.008^{*}$ \\
\hline Cerebrovascular disease & $21(80.8)$ & $57(46.7)$ & $0.002^{*}$ \\
\hline Chronic respiratory disease & $6(23.1)$ & $9(7.4)$ & $0.040^{*}$ \\
\hline Chronic kidney disease & $13(50.0)$ & $33(27.0)$ & $0.022^{*}$ \\
\hline Diabetes mellitus & $9(34.6)$ & $44(36.1)$ & 0.889 \\
\hline Malignant tumor & $4(15.4)$ & $44(36.1)$ & $0.041^{*}$ \\
\hline Anemia & $26(100.0)$ & $87(71.3)$ & $0.002^{*}$ \\
\hline Hypoalbuminemia & $26(100.0)$ & $98(80.3)$ & $0.029^{*}$ \\
\hline Lung infection & $22(84.6)$ & $61(50.0)$ & $0.001^{*}$ \\
\hline Urinary tract infection & $12(46.2)$ & $62(50.8)$ & 0.666 \\
\hline Abdominal infection & $5(19.2)$ & $14(11.5)$ & 0.453 \\
\hline Tracheotomy & $12(46.2)$ & $21(17.2)$ & $0.001^{*}$ \\
\hline Liver dysfunction & $13(50.0)$ & $24(19.7)$ & 0.001 \\
\hline \multicolumn{4}{|l|}{ Invasive surgery before infection } \\
\hline Mechanical ventilation & $18(69.2)$ & $12(9.8)$ & $<0.001^{*}$ \\
\hline Central venous catheterization & $19(73.1)$ & $28(23.0)$ & $<0.001^{*}$ \\
\hline Indwelling urethral catheterization & $25(96.2)$ & $45(36.9)$ & $<0.001^{*}$ \\
\hline \multicolumn{4}{|l|}{ Sources of bloodstream infection } \\
\hline Catheter related & $10(38.5)$ & $23(18.9)$ & $0.029^{*}$ \\
\hline Respiratory system & $11(42.3)$ & $26(21.3)$ & $0.025^{*}$ \\
\hline Urinary tract infection & $0(0)$ & $42(34.4)$ & $<0.001^{*}$ \\
\hline Others & $5(19.2)$ & $31(25.4)$ & 0.505 \\
\hline Prior use of broad-spectrum antibiotics within 30 days & $25(96.2)$ & $39(32.0)$ & $<0.001^{*}$ \\
\hline Combined fungal infection & $10(38.5)$ & $10(8.2)$ & $<0.001^{*}$ \\
\hline Prior use of glucocorticoids within 30 days & $8(30.8)$ & $11(9.0)$ & $0.007^{*}$ \\
\hline Total length of hospital stay & $25.4 \pm 11.1$ & $21.0 \pm 12.7$ & 0.102 \\
\hline 30-Day mortality rate & $17(65.4)$ & $21(17.2)$ & $<0.001^{*}$ \\
\hline 48-Hour mortality rate & $6(23.1)$ & $3(2.5)$ & $<0.001^{*}$ \\
\hline
\end{tabular}

ICU: intensive care unit; ${ }^{*} P<0.05$.

TABLE 2: Analysis of risk factors for CRE bloodstream infections (logistic regression analysis).

\begin{tabular}{|c|c|c|c|}
\hline Risk factor & OR value & $95 \% \mathrm{CI}$ & $P$ value \\
\hline Indwelling urethral catheterization & 11.40 & 1.05 to 124.18 & 0.046 \\
\hline Admission to the ICU & 9.42 & 2.14 to 41.50 & 0.003 \\
\hline Respiratory system source & 8.95 & 1.73 to 46.38 & 0.009 \\
\hline Prior use of broad-spectrum antibiotics within 30 days & 11.25 & 1.19 to 106.79 & 0.035 \\
\hline
\end{tabular}

OR: odds ratio; 95\% CI: 95\% confidence interval; respiratory system source: bloodstream infection with Enterobacteriaceae from the respiratory system.

The results of this study on risk factors for death due to bloodstream infections with Enterobacteriaceae showed that lung infections, abdominal infections, central venous catheterization, and hormone use within 30 days were all independent risk factors for death due to bloodstream infections with Enterobacteriaceae. The study results showed that the mortality rate was significantly higher in the CRE group than in the non-CRE group. The high mortality 
TABLE 3: Univariate analysis for predictors of mortality caused by bloodstream infections with Enterobacteriaceae.

\begin{tabular}{|c|c|c|c|}
\hline \multirow{2}{*}{ Clinical characteristics } & \multicolumn{2}{|c|}{ Bloodstream infections with Enterobacteriaceae, $n$ (\%) } & \multirow{2}{*}{$P$ value } \\
\hline & Survival group $(n=110)$ & Mortality group $(n=38)$ & \\
\hline Gender (male) & $55(50.0)$ & $20(52.6)$ & 0.780 \\
\hline Age & $67.5 \pm 20.0$ & $73.9 \pm 15.9$ & 0.075 \\
\hline Community acquired & $28(25.5)$ & $2(5.3)$ & $0.008^{*}$ \\
\hline Admission to the ICU & $10(9.1)$ & $20(52.6)$ & $<0.001^{*}$ \\
\hline Long-term bedbound & $50(45.5)$ & $30(78.9)$ & $<0.001^{*}$ \\
\hline Medical institution admission history within 3 months & $69(62.7)$ & $23(60.5)$ & 0.809 \\
\hline \multicolumn{4}{|l|}{ Underlying diseases } \\
\hline Cardiovascular disease & $51(46.4)$ & $22(57.9)$ & 0.220 \\
\hline Cerebrovascular disease & $54(49.1)$ & $24(63.2)$ & 0.134 \\
\hline Chronic respiratory disease & $12(10.9)$ & $3(7.9)$ & 0.827 \\
\hline Chronic kidney disease & $30(27.3)$ & $16(42.1)$ & 0.089 \\
\hline Diabetes mellitus & $38(34.5)$ & $15(39.5)$ & 0.585 \\
\hline Malignant tumor & $37(33.6)$ & $11(28.9)$ & 0.594 \\
\hline Anemia & $77(70.0)$ & $36(94.7)$ & $0.002^{*}$ \\
\hline Hypoalbuminemia & $87(79.1)$ & $37(97.4)$ & $0.008^{*}$ \\
\hline Lung infection & $53(48.2)$ & $30(78.9)$ & $0.001^{*}$ \\
\hline Urinary tract infection & $60(54.5)$ & $14(36.8)$ & 0.060 \\
\hline Abdominal infection & $9(8.2)$ & $10(26.3)$ & $0.009^{*}$ \\
\hline Tracheotomy & $22(20.0)$ & $11(28.9)$ & 0.253 \\
\hline Liver dysfunction & $20(18.2)$ & $17(44.7)$ & $0.001^{*}$ \\
\hline \multicolumn{4}{|l|}{ Invasive surgery before infection } \\
\hline Mechanic ventilation & $10(9.1)$ & $20(52.6)$ & $<0.001^{*}$ \\
\hline Central venous catheterization & $20(18.2)$ & $27(71.1)$ & $<0.001^{*}$ \\
\hline Indwelling urethral catheterization & $41(37.3)$ & $29(76.3)$ & $<0.001^{*}$ \\
\hline \multicolumn{4}{|l|}{ Sources of bloodstream infection } \\
\hline Catheter related & $19(17.3)$ & $14(36.8)$ & $0.012^{*}$ \\
\hline Respiratory system & $25(22.7)$ & $12(31.6)$ & 0.277 \\
\hline Urinary tract & $39(35.5)$ & $3(7.9)$ & $0.001^{*}$ \\
\hline Others & $27(24.5)$ & $9(23.7)$ & 0.915 \\
\hline Prior use of broad-spectrum antibiotics within 30 days & $36(32.7)$ & $28(73.7)$ & $<0.001^{*}$ \\
\hline Combined fungal infection & $9(8.2)$ & $11(28.9)$ & $<0.001^{*}$ \\
\hline Prior use of glucocorticoids within 30 days & $4(3.6)$ & $15(39.5)$ & $0.007^{*}$ \\
\hline CRE infection & $9(8.2)$ & $17(44.7)$ & $<0.001^{*}$ \\
\hline Total length of hospital stay & $21.4 \pm 13.2$ & $22.7 \pm 10.4$ & 0.528 \\
\hline
\end{tabular}

CRE: carbapenem-resistant Enterobacteriaceae; ${ }^{*} P<0.05$.

TABLE 4: Logistic regression analysis of risk factors for mortality in patients with Enterobacteriaceae bloodstream infections.

\begin{tabular}{lccc}
\hline Risk factor & OR value & $95 \%$ CI & $P$ value \\
\hline Lung infection & 6.62 & 1.86 to 23.61 & 0.004 \\
Abdominal infection & 11.15 & 2.54 to 48.92 & 0.001 \\
Central venous catheterization & 5.25 & 1.84 to 14.94 & 0.002 \\
Prior use of glucocorticoids within 30 days & 22.40 & 4.07 to 123.38 & $<0.001$ \\
\hline
\end{tabular}

OR: odds ratio; 95\% CI: 95\% confidence interval.

associated with CRE bloodstream infections was partially attributed to host conditions, such as endogenous infections and invasive surgery. Additionally, the mortality rate was associated with a lack of timely and accurate antibiotic treatment for infection with drug-resistant bacterial strains or even no antibiotic treatment [10]. XDR strains accounted for $23 \%$ of the 26 cases of CRE infections in this study, which was similar to the $28 \%$ rate of XDR bacterial strains isolated from 
CRE infections reported in the literature [8]. This result indicated that approximately one-fourth of CRE infections might be $\mathrm{XDR}$, which is associated with a high mortality rate.

During the analysis of mortality-related risk factors in patients with bloodstream Enterobacteriaceae infections in this study, CRE infection was a relevant but not an independent risk factor, which might be associated with the low overall number of cases.

In summary, CRE bloodstream infections have a high mortality rate, and treatment has poor clinical efficacy. The use of broad-spectrum antibiotics, indwelling urethral catheterization, admission to the ICU, and infection from the respiratory system were independent risk factors for CRE bloodstream infections. Lung infections, abdominal infections, hormone use, and central venous catheterization were independent risk factors for death in patients with Enterobacteriaceae bloodstream infections. Therefore, the standard use of antibiotics, minimization of invasive operations, use of strict aseptic manipulation, and strengthening of handhygiene awareness of healthcare workers may be effective measures for the prevention of CRE bloodstream infections.

\section{Conflicts of Interest}

The authors declare that there are no conflicts of interest.

\section{References}

[1] K. B. Laupland and D. L. Church, "Population-based epidemiology and microbiology of community-onset bloodstream infections," Clinical Microbiology Reviews, vol. 27, no. 4, pp. 647-664, 2014.

[2] R. Picot-Guéraud, P. Batailler, Y. Caspar, A. Hennebique, and M. R. Mallaret, "Bacteremia caused by multidrug-resistant bacteria in a French university hospital center: 3 years of collection," American Journal of Infection Control, vol. 43, no. 9, pp. 960-964, 2015.

[3] F. Hu, D. Zhu, F. Wang et al., "CHINET 2014 surveillance of bacterial resistance in China," Chinese Journal of Infection and Chemotherapy, vol. 1, pp. 401-410, 2015.

[4] M. E. Falagas, G. S. Tansarli, D. E. Karageorgopoulos, and K. Z. Vardakas, "Deaths attributable to carbapenem-resistant Enterobacteriaceae infections," Emerging Infectious Diseases, vol. 20, no. 7, pp. 1170-1175, 2014.

[5] F. Cockerill, M. Wikler, K. Bush et al., Performance Standards for Antimicrobial Susceptibility Testing: Twentieth Informational Supplement, M100-S20, Clinical Laboratory Standards Institute (CLSI), Wayne, PA, USA, 2010.

[6] A. P. Magiorakos, A. Srinivasan, R. B. Carey et al., "Multidrugresistant, extensively drug-resistant and pandrug-resistant bacteria: an international expert proposal for interim standard definitions for acquired resistance," Clinical Microbiology and Infection, vol. 18, no. 3, pp. 268-281, 2012.

[7] M. Ferrall, New Definition of Bedridden in a RCFE, RCFE Legislative Updates and Public Policy, 2011.

[8] S. Lofmark, K. Sjöström, B. Mäkitalo, P. Edquist, K. Tegmark Wisell, and C. G. Giske, "Carbapenemase-producing Enterobacteriaceae in Sweden 2007-2013: experiences from seven years of systematic surveillance and mandatory reporting," Drug Resistance Updates, vol. 20, pp. 29-38, 2015.

[9] J. C. Gallagher, S. Kuriakose, K. Haynes, and P. Axelrod, "Case-case-control study of patients with carbapenem- resistant and third-generation-cephalosporin-resistant Klebsiella pneumoniae bloodstream infections," Antimicrobial Agents and Chemotherapy, vol. 58, no. 10, pp. 5732-5735, 2014.

[10] M. Falcone, A. Vena, M. L. Mezzatesta et al., "Role of empirical and targeted therapy in hospitalized patients with bloodstream infections caused by ESBL-producing Enterobacteriaceae," Annali di Igiene: Medicina Preventiva e di Comunita, vol. 26, no. 4, pp. 293-304, 2014. 


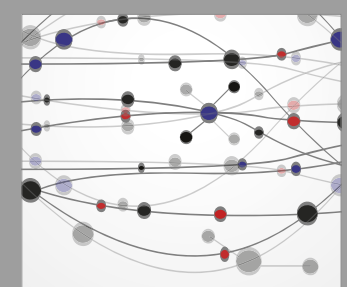

The Scientific World Journal
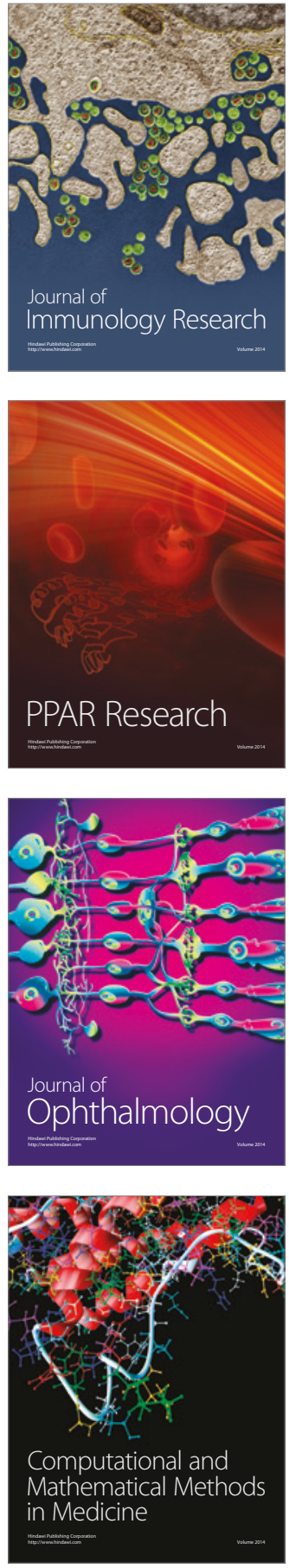

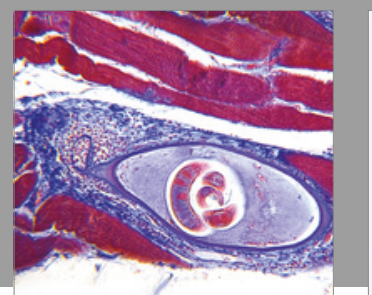

Gastroenterology Research and Practice
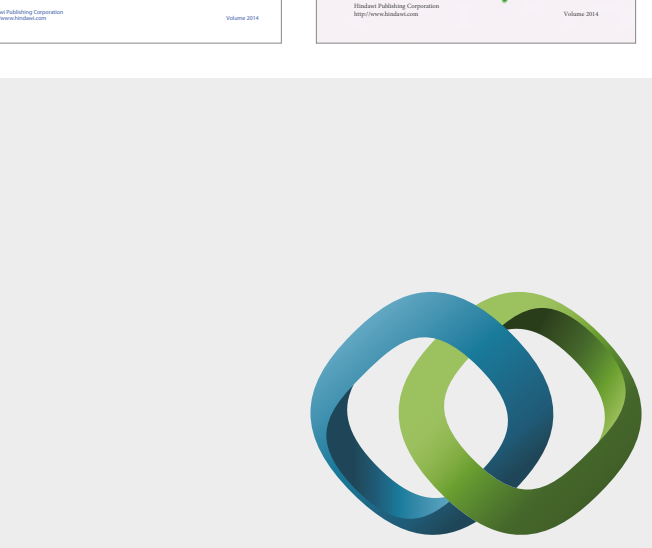

\section{Hindawi}

Submit your manuscripts at

https://www.hindawi.com
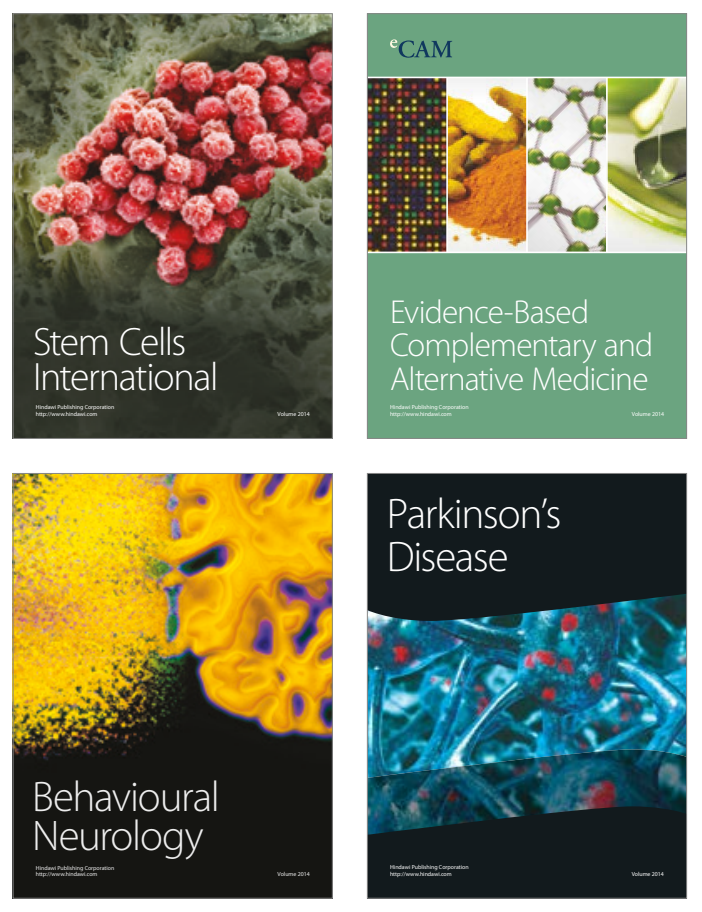
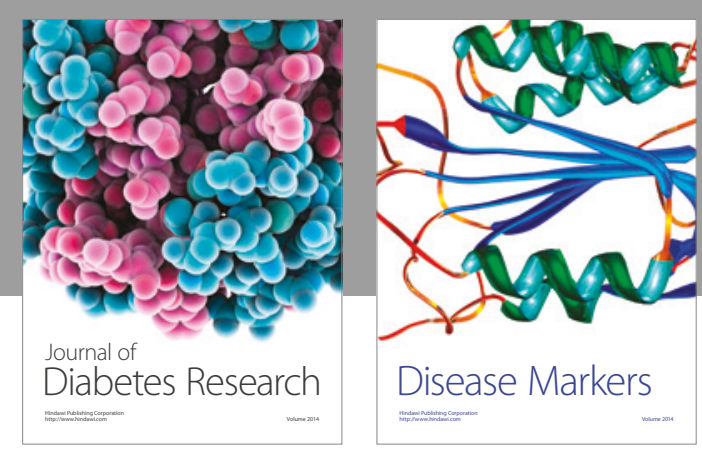

Disease Markers
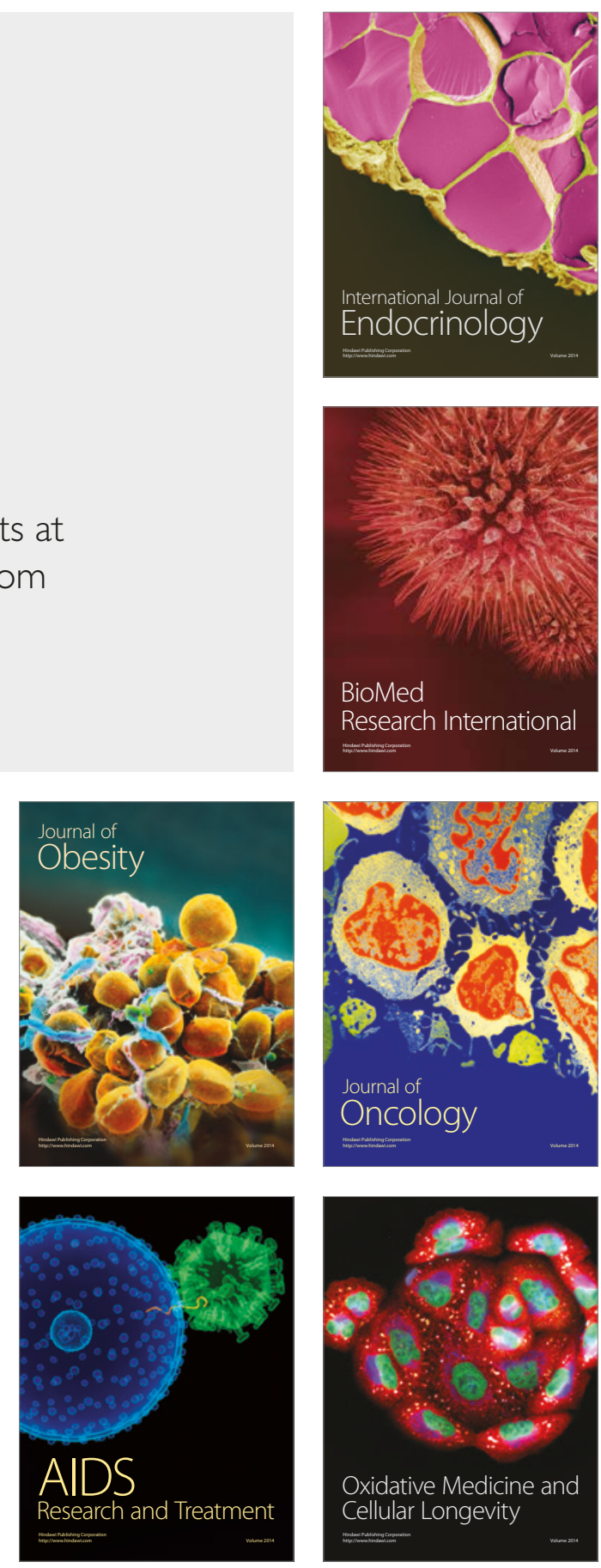